\title{
Obligation towards medical errors disclosure at a tertiary care hospital in Dubai, UAE
}

\author{
Ashraf Ahmad Zaghloul ${ }^{\mathrm{a}, \mathrm{b}, *}$, Syed Azizur Rahman ${ }^{\mathrm{c}, \mathrm{d}}$ and Nagwa Younes Abou El-Enein ${ }^{\mathrm{e}}$ \\ ${ }^{a}$ Health Policy, Planning and Administration, Department of Public Health Administration and \\ Behavioural Sciences, High Institute of Public Health, University of Alexandria, Alexandria, Egypt \\ ${ }^{\mathrm{b}}$ Chair of Health Services Administration Department, College of Health Science, University \\ of Sharjah, Sharjah, United Arab Emirates \\ ${ }^{\mathrm{c}}$ Health Services Administration, College of Health Sciences, University of Sharjah, Sharjah, \\ United Arab Emirates \\ ${ }^{\mathrm{d}}$ School of Population and Public Health, University of British Columbia, BC, Canada \\ ${ }^{\mathrm{e}}$ Health Policy, Planning and Administration, High Institute of Public Health, University \\ of Alexandria, Alexandria, Egypt
}

\begin{abstract}
.
OBJECTIVE: The study aimed to identify healthcare providers' obligation towards medical errors disclosure as well as to study the association between the severity of the medical error and the intention to disclose the error to the patients and their families.

DESIGN: A cross-sectional study design was followed to identify the magnitude of disclosure among healthcare providers in different departments at a randomly selected tertiary care hospital in Dubai.

SETTING AND PARTICIPANTS: The total sample size accounted for 106 respondents. Data were collected using a questionnaire composed of two sections namely; demographic variables of the respondents and a section which included variables relevant to medical error disclosure.

RESULTS: Statistical analysis yielded significant association between the obligation to disclose medical errors with male healthcare providers $\left(X^{2}=5.1\right)$, and being a physician $\left(X^{2}=19.3\right)$. Obligation towards medical errors disclosure was significantly associated with those healthcare providers who had not committed any medical errors during the past year $\left(X^{2}=9.8\right)$, and any type of medical error regardless the cause, extent of harm $\left(\mathrm{X}^{2}=8.7\right)$. Variables included in the binary logistic regression model were; status $(\operatorname{Exp} \beta($ Physician $)=0.39,95 \%$ CI 0.16-0.97), gender $($ Exp $\beta($ Male $)=4.81,95 \%$ CI 1.84-12.54), and medical errors during the last year $(\operatorname{Exp} \beta$ (None) $=2.11,95 \%$ CI $0.6-2.3)$.

CONCLUSION: Education and training of physicians about disclosure conversations needs to start as early as medical school. Like the training in other competencies required of physicians, education in communicating about medical errors could help reduce physicians' apprehension and make them more comfortable with disclosure conversations.
\end{abstract}

Keywords: Obligation, medical error, disclosure

\section{Introduction}

Several international reports pointed out to the prevalence of medical errors and its implications for health care system. In the United States, medical errors are ranked as the eighth leading cause of

\footnotetext{
*Address for correspondence: Ashraf Ahmad Zaghloul, MD, DrPH, Professor of Health Policy, Planning \& Administration, Department of Public Health Administration and Behavioural Sciences, High Institute of Public Health, 165, El-Horreya Avenue, Postal Code Alexandria 21561, University of Alexandria, Alexandria, Egypt and Associate Professor and Chair of Health Services Administration Department, College of Health Sciences, University of Sharjah, Sharjah, Postal Code Sharjah 27272, United Arab Emirates. Tel.: +971 5011539 77; E-mail: zaghloul.ashraf@ gmail.com.
} 
death. A total of 6.3 million medical injuries were reported in 2008, and approximately 1.5 million were associated with a medical error. The estimated average total cost per error was approximately 13,000 dollars. In Australia (2003), about 1\% of all hospital patients suffered from an adverse health event as a result of a medication error [1,2]. Medical error is a non-intentional harm from a treatment or a practice that is directed initially to improve patients' health status. Since the early 1990s, there has been a growing awareness that patients are subject to injuries that could be prevented as a direct consequence of health care [3,4]. Preventable injuries damage patient and family trust in the provider and the institution $[5,6]$. Error disclosure is an effective technique to restore the lost trust. Medical errors is a real problem in healthcare services, most doctors do not report errors formally and even do not learn from their mistakes. The disclosure of medical errors in healthcare settings is considered as a vital part of ongoing activities to improve patient safety and the quality of care [7].

Ethical obligations and patient safety principles support prompt disclosure of harmful medical errors. In USA, the Patient Safety and Quality Improvement Act on July, 29, 2005 discussed an ethical and professional consensus that obligated doctors and nurses to disclose medical errors [8]. However, McDonnell and Guenther (2008) stated that fear of malpractice liability is a barrier to physician disclosure and that, healthcare providers fear disclosure will invite new claims or complicate the medical error incident and therefore acts as an inhibiting impulse to disclose [9].

Disclosure and its elements are emphasized in ethical and professional guidelines. Since 2001, The Joint Commission has required hospitals to disclose all unexpected outcomes. Disclosure allows a patient to obtain timely and appropriate treatment to correct problems; additionally, it enables better informed consent for any further treatment that may be required $[10,11]$.

The literature reveals that, after a medical error, the patients want a clear statement that an error has occurred and an explicit apology. However, there is a gap between the patients' desire for information after iatrogenic injuries and the actual practice of disclosure by healthcare providers [12-14].

Studies have revealed that physicians are committed to telling their patients the truth; however, the fear of litigation, the culture of medicine, and the inherent psychological difficulties in facing one's mistakes limit their actual disclosure practices. In a study, surgeons used the words error or mistake in only $57 \%$ of their disclosure conversations, took responsibility for the error in $65 \%$ of encounters, and offered a verbal apology in 47\%. A study also revealed that the likelihood of disclosure is dependent on the severity of the outcome of the error, with $74 \%$ of physicians agreeing that disclosing a serious error would be very difficult. Trainees participating in a study in internal medicine departments at two academic medical centers chose their words carefully to avoid being explicit about the error; likewise, only $50 \%$ used statements that explicitly stated that an error had occurred, and $46 \%$ agreed to offer an explicit apology rather than a general expression of regret [15-17].

The literature remains deficient in revealing the factors that facilitate or hinder health professionals in disclosing medical errors to their patients and the patients' relatives. Organizational cultures governing the context of patient safety in hospitals are responsible for governing health professional disclosure or nondisclosure of the medical accident. The current study aimed to healthcare providers' obligation towards medical errors disclosure as well as to study the association between relevant characteristics of the medical error and the intention to disclose the error to the patients and their families.

\section{Methods}

Study Design: Non-experimental, cross-sectional study design.

Study Setting: A list of all the private hospitals present in Dubai, United Arab Emirates (UAE) was obtained (23 private hospitals). One private tertiary hospital in Dubai, UAE was selected randomly using the bowl technique to conduct the study. 
Study Population: All health care professionals working at the private hospital, at the time of the study were included in the study sample.

Sample Size: The total sample size accounted for 204 participants. A total of 106 questionnaires were complete and eligible for further statistical analysis.

Data Collection Tool: The questionnaire was developed after reviewing the relevant literature on medical disclosure. Statements from the literature pertinent to the aim of the study were developed to formulate the final version of the questionnaire $[5,6,18,19]$. The questionnaire was pilot tested among 8 health care providers (physicians and nurses) who had at least 10 years of experience with their relevant practice. Feedback was collected, and minor modifications to the questionnaire were made after the pilot study. The questionnaire included two sections. Section 1, demographic data which entailed variables; age, gender, marital status, type of practice, years of experience. Section 2, obligation towards disclosure of medical errors which entailed statements; times committed medical errors throughout career, number of medical errors during the last year, outcome of medical error, disclosure to patient/family, extent of medical error.

\subsection{Statistical analysis}

Complete questionnaires were coded and used for statistical analysis. Statistical analyses were performed using Statistical Package for the Social Sciences (SPSS) software (v. 21.0; IBM Corporation, Armonk, NY, USA). Data coding and data entry were performed and variables with missing data were excluded from further statistical analysis. Tabulation of the categorical variables was presented in the form of number and percent along with the relevant test of significance of association. Demographic, exposure variables, and confounders were screened for inclusion in the multivariate binary logistic regression model. Candidate variables with values of $p<0.05$ were included in the logistic regression model. Exposure and confounder variables were categorized and binary-encoded $(0=$ reference group; $1=$ contrast group). Statistical tests were two-tailed adjusted, and values of $p<0.05$ were considered to be statistically significant.

\subsection{Ethical considerations}

The research proposal was submitted for scientific and ethical approval through the Medical Research Committee at the private hospital. The study was conducted throughout the month of April 2015. On the cover page of the questionnaire, a statement was included stating that responding to the items of the questionnaire was considered an implicit approval of participating in the study. The participants were informed of the option to refrain from joining the study or to withdraw at any phase of the data collection. The participants were informed that all data collected would be kept confidential and would be deidentified and destroyed after publication of the results of the study.

\section{Results}

Table 1, shows the association between demographic variables and the obligation towards medical errors disclosure. Two variables were significantly associated with medical errors disclosure, namely; gender and practice. Male healthcare providers were significantly associated with the obligation to report medical errors than female healthcare providers $\left(\mathrm{X}^{2}=5.1\right)$. Physicians were significantly associated with medical errors disclosure than the rest of the members of the healthcare team $\left(\mathrm{X}^{2}=19.3\right)$.

Table 2, shows the association between obligation to disclose medical errors with disclosure items. Two items were significantly associated with medical errors disclosure, namely; medical errors committed during the last year, and type of medical error disclosed. Obligation towards medical errors 
Table 1

Demographic variables by attitude and practice towards medical errors disclosure (Dubai, 2015)

\begin{tabular}{|c|c|c|c|c|c|}
\hline & \multicolumn{5}{|c|}{ Obligation towards medical errors disclosure } \\
\hline & \multicolumn{2}{|c|}{ Yes } & \multicolumn{2}{|c|}{ No } & \multirow[b]{2}{*}{$\mathrm{X}^{2}$} \\
\hline & No. (56) & $\%$ & No. (50) & $\%$ & \\
\hline \multicolumn{6}{|l|}{ Age } \\
\hline Less than 30 years & 16 & 28.6 & 18 & 36.0 & 1.7 \\
\hline 30 to less than 40 & 30 & 53.6 & 24 & 48.0 & \\
\hline $40+$ & 10 & 17.8 & 8 & 16.0 & \\
\hline \multicolumn{6}{|l|}{ Gender } \\
\hline Male & 36 & 64.3 & 20 & 40.0 & $5.1^{*}$ \\
\hline Female & 20 & 35.7 & 30 & 60.0 & \\
\hline \multicolumn{6}{|l|}{ Marital Status } \\
\hline Single & 14 & 25.9 & 20 & 40.0 & 2.4 \\
\hline Married & 34 & 63.0 & 24 & 48.0 & \\
\hline Divorced/widowed & 6 & 11.1 & 6 & 12.0 & \\
\hline \multicolumn{6}{|l|}{ Practice } \\
\hline Physician & 26 & 46.4 & 8 & 16.0 & $19.3^{*}$ \\
\hline Nurse & 8 & 14.3 & 6 & 12.0 & \\
\hline Lab. Technologist & 6 & 10.7 & 22 & 44.0 & \\
\hline Radiologist & 12 & 21.4 & 10 & 20.0 & \\
\hline Pharmacist & 4 & 7.1 & 4 & 8.0 & \\
\hline \multicolumn{6}{|l|}{ Experience } \\
\hline Less than 5 years & 20 & 35.7 & 18 & 36.0 & 0.11 \\
\hline 5 to less than 10 years & 18 & 32.1 & 16 & 32.0 & \\
\hline $10+$ & 18 & 32.1 & 16 & 32.0 & \\
\hline
\end{tabular}

${ }^{*} P$ value $<0.05$

disclosure was significantly associated with those healthcare providers who had not committed any medical errors during the past year $\left(X^{2}=9.8\right)$. Whereas, obligation towards disclosure was significantly associated with any type of medical error regardless the cause, extent of harm $\left(\mathrm{X}^{2}=8.7\right)$.

Table 3 shows the significant variables yielded from the statistical analysis and included in the binary logistic regression model. The variables namely; status $(\operatorname{Exp} \beta($ Physician $)=0.39,95 \%$ CI 0.16-0.97), gender $(\operatorname{Exp} \beta($ Male $)=4.81,95 \%$ CI 1.84-12.54), and medical errors during the last year $(\operatorname{Exp} \beta$ $($ None $)=2.11,95 \%$ CI $0.6-2.3)$.

\section{Discussion}

Much attention has focused on the management of medical mistakes in recent years. Currently, there is a consensus that disclosing medical mistakes is advantageous for patients, clinicians, and medical organizations in reducing the number of medical malpractice suits and increasing patient satisfaction which relevant more for private healthcare services. Although a large number of interventions have been developed to facilitate mistake disclosures, evidence demonstrates that clinicians and healthcare providers have been slow to adopt those practices. Therefore, one of the problems may be the need for an alternative, theory-based model that provides a tool to guide initial conversations with patients after a mistake, which can be followed up with additional details. Thus, a more directed set of strategies may provide impetus for physicians to make a disclosure closer to the mistake event and to do so effectively [17, 20, 21]. More emphasis is directed to female healthcare providers who in this study 
Table 2

Association between obligation to disclose medical errors with disclosure items (Dubai, 2015)

\begin{tabular}{|c|c|c|c|c|c|}
\hline & \multicolumn{5}{|c|}{ Obligation towards medical errors disclosure } \\
\hline & \multicolumn{2}{|c|}{ Yes } & \multicolumn{2}{|c|}{ No } & \multirow[b]{2}{*}{$X^{2}$} \\
\hline & No. $(56)$ & $\%$ & No. $(50)$ & $\%$ & \\
\hline \multicolumn{6}{|c|}{ Number of committed medical errors throughout experience } \\
\hline Once & 22 & 39.3 & 18 & 36.0 & 1.7 \\
\hline Twice & 20 & 35.7 & 10 & 20.0 & \\
\hline Three+ & 14 & 25.0 & 22 & 44.0 & \\
\hline \multicolumn{6}{|l|}{ Medical errors during the last year } \\
\hline None & 36 & 64.3 & 16 & 32.0 & $9.8^{*}$ \\
\hline One or more & 20 & 35.7 & 34 & 68.0 & \\
\hline \multicolumn{6}{|l|}{ Outcome of medical error } \\
\hline Harmless & 38 & 67.9 & 40 & 80.0 & 2.8 \\
\hline Temporary disability & 4 & 7.1 & 4 & 8.0 & \\
\hline Permanent disability & 4 & 7.1 & 2 & 4.0 & \\
\hline Death & 10 & 18.9 & 4 & 8.0 & \\
\hline \multicolumn{6}{|l|}{ Patient/family disclosed about the medical error } \\
\hline Yes & 28 & 50.0 & 8 & 16.0 & 0.5 \\
\hline No & 28 & 50.0 & 42 & 84.0 & \\
\hline \multicolumn{6}{|l|}{ Type of medical error disclosed } \\
\hline Any medical error & 32 & 57.1 & 12 & 24.0 & $8.7^{*}$ \\
\hline A medical error that causes moderate/ major harm & 18 & 32.1 & 18 & 36.0 & \\
\hline A major harm only & 4 & 7.1 & 16 & 32.0 & \\
\hline A medical error should not be disclosed & 2 & 3.6 & 4 & 8.0 & \\
\hline
\end{tabular}

${ }^{*} P$ value $<0.05$

Table 3

Logistic regression of predictors of obligation towards medical errors disclosure (Dubai, 2015)

\begin{tabular}{lccccc}
\hline Variable & B & SE & Exp (B) & Significance & $95 \%$ CI \\
\hline Constant & -0.6 & 0.48 & 0.53 & & \\
Practice (Physician) & -0.9 & 0.46 & 0.39 & 0.04 & $0.16-0.97$ \\
Gender (Male) & 1.5 & 0.48 & 4.81 & 0.001 & $1.84-12.54$ \\
Medical errors during the last year (None) & 1.2 & 1.1 & 2.11 & 0.03 & $0.6-2.3$ \\
\hline
\end{tabular}

might have been under the influence of losing the job in an Arabian Gulf country and/or the strict regulations followed in the private sector towards quality and patient safety with its reflections on the hospital image. Within the same context, not only physicians but strategies should be directed towards empowerment of other healthcare team members when facing the alternatives and consequences of a medical error [22].

Hospital administrators will also need to develop training programs and workshops on the disclosure of adverse events and errors and to provide support and counseling to surgeons other health care professionals to help them cope with their mistakes. All hospitals and surgical departments should establish an error disclosure unit with the responsibility of supporting full disclosure. The unit could develop a written institutional protocol including an explicit statement that there was an error, details of what went wrong, and why the adverse event or error occurred. 
An anonymous reporting system should be established to collect and analyze information about adverse events and errors, emphasizing relevant information about both the processes of care and the consequences of these actions. It has been stated by Leappe that support systems need to be developed for all parties. Patients need help after an event, including after discharge from the hospital, and there is also a need to provide support and "just-in-time training" to help the physician communicate with the patient after the event; additionally, professional and peer support systems must be developed [5].

Physicians and risk managers are particularly concerned that disclosure may increase the chances of being sued, which is why many physicians never admit their mistakes. One study showed that full disclosure and the acceptance of responsibility were related to a reduction in intention to sue, which was also influenced by the severity of the outcomes as determinants for future actions by patients and their families [17]. It was found that disclosure had a protective effect of $7.1 \%$ against seeking legal advice. Another study noted that, in severe adverse outcomes, an honest, empathic, and accountable approach decreased patients' support for strong physician sanctions by 59\% [14].

Health care providers all over the world have a similar prospectus toward disclosure as well as similar beliefs and inherent norms. Educators at medical schools have the task of improving a trainee's approaches to disclosure. The first step is to start as early as years 2 and 3 of medical school. One study concluded that medical school students show willingness toward disclosure at their earlier stages of medical education. This suggests that educators have an important window of opportunity in which to inculcate positive disclosure attitudes early in the medical education process [23, 24].

\section{Conclusion}

Education and training of physicians about disclosure conversations needs to start as early as medical school. Like the training in other competencies required of physicians, education in communicating about medical errors could help reduce physicians' apprehension and make them more comfortable with disclosure conversations.

\subsection{Limitations}

The response rate and incomplete questionnaire items, as well as the small sample size surveyed pose implications on the generalizability of the results of the study. Respondent bias owing to the sensitivity of the issue tackled in the study as some participants were uncomfortable to answer such sensitive questions about admitting the commitment of a medical error. The education about the importance of participation in research for community benefits requires further commitment and improvement.

\section{Conflict of interest}

The authors declare no conflicts of interest in this work either financial or scientific with any organization. This research received no specific grant from any funding agency in the public, commercial, or not-for-profit sectors. The authors alone are responsible for the content and writing of the paper.

\section{Funding}

The research is funded by the researchers throughout all the stages of the study. 


\section{References}

[1] Wilson J, McCaffrey R. Disclosure of Medical Errors to Patients. Medsurg Nurs. 2005;14:319-23.

[2] Runciman W, Roughead E, Semple S, et al. Adverse drug events and medication errors in Australia. Int J Qual Health Care. 2003;15(Suppl 1):i49-59.

[3] Levinson W, Gallagher TH. Disclosing medical errors to patients: A status report in 2007. CMAJ 2007;177:265-7. doi:10.1503/cmaj.061413

[4] Sorensen R, Iedema R, Piper D, et al. Disclosing clinical adverse events to patients: Can practice inform policy? Health Expect. 2010;13:148-59. doi:10.1111/j.1369-7625.2009.00569.x. Epub 2009 Oct 5.

[5] O'Connor E, Coates HM, Yardley IE, et al. Disclosure of patient safety incidents: A comprehensive review. Int J Qual Health Care. 2010;22:371-9. doi:10.1093/intqhc/mzq042

[6] Leape LL. Full disclosure and apology—an idea whose time has come. Physician Exec. 2006;32:16-8.

[7] Kroll L, Singleton A, Collier J, et al. Learning not to take it seriously: Junior doctors' accounts of error. Med Educ. 2008;42:982-90. doi:10.1111/j.1365-2923.2008.03151.x

[8] Clinton H, Obama B. Making patient safety the centerpiece of medical liability reform. N Engl J Med. 2006;354:2205-8.

[9] McDonnell W, Guenther E. Narrative review: Do state laws make it easier to say "I'm sorry?" Ann Intern Med. 2008;149:811-5. doi:10.7326/0003-4819-149-11-200812020-00007

[10] Snyder L, Leffler C. Ethics and Human Rights Committee, American College of Physicians. Ethics manual: Fifth edition. Ann Intern Med. 2005;142:560-82.

[11] American Board of Internal Medicine, American College of Physicians-American Society of Internal Medicine, European Federation of Internal Medicine. Medical professionalism in the new millennium: A physician charter. Ann Intern Med. 2002; 136:243-6.

[12] Mazor KM, Goff SL, Dodd K, et al. Understanding patients' perceptions of medical errors. J Commun Healthc. 2009;2:34-46.

[13] Bismark M, Dauer E, Paterson R, et al. Accountability sought by patients following adverse events from medical care: The New Zealand experience. CMAJ. 2006;175:889-4.

[14] Wu AW, Huang IC, Stokes S, et al. Disclosing medical errors to patients: It's not what you say, it's what they hear. J Gen Intern Med. 2009;24:1012-7. doi:10.1007/s11606-009-1044-3

[15] Gallagher TH, Waterman AD, Garbutt JM, et al. US and Canadian physicians' attitudes and experiences regarding disclosing errors to patients. Arch Intern Med. 2006;166:1605-11.

[16] White AA, Bell SK, Krauss MJ, et al. How trainees would disclose medical errors: Educational implications for training programmes. Med Educ. 2011;45:372-80. doi:10.1111/j.1365-2923.2010.03875.x

[17] Zaghloul AA, Elsergany M, Mosallam R. A Measure of barriers toward medical disclosure among health professionals in the United Arab Emirates. J Patient Saf. Epub ahead of print March 23, 2015. March 23, 2015 doi: 10.1097/PTS.0000000000000166

[18] Wu AW, Cavanaugh TA, McPhee SJ, et al. To tell the truth: Ethical and practical issues in disclosing medical mistakes to patients. J Gen Intern Med. 1997;12:770-5.

[19] Blendon RJ, DesRoches CM, Brodie M, et al. Views of practicing physicians and the public on medical errors. N Engl J Med. 2002; 347:1933-40.

[20] Kaldjian LC, Jones EW, Rosenthal GE. Facilitating and impeding factors for physicians' error disclosure: A structured literature review. Jt Comm J Qual Patient Saf. 2006;32:188-98.

[21] Helmchen LA, Richards MR, McDonald TB. How does routine disclosure of medical error affect patients' propensity to sue and their assessment of provider quality? Evidence from survey data. Med Care. 2010;48;955-61. doi:10.1097/MLR.0b013e3181eaf84d

[22] Ushie BA, Salami KK, Jegede AS, et al. Patients' knowledge and perceived reactions to medical errors in a tertiary health facility in Nigeria. African Health Sciences. 2013;13:820-8.

[23] Ibrahim A, Garba ES, Asuku ME. Challenges in disclosure of adverse events and errors in surgery; perspectives from sub-Saharan Africa. Pan Afr Med J. 2012;12:82.

[24] White AA, Bell SK, Krauss MJ, et al. How trainees would disclose medical errors: Educational implications for training programmes. Med Educ. 2011;45:372-80. doi:10.1111/j.1365-2923.2010.03875.x 